\title{
Association between Morningness-Eveningness and Defense Mechanisms in Community-Dwelling Korean Adults
}

\author{
Jichul Kim, Somin Kim, Sehyun Jeon, and Seog Ju Kim \\ Department of Psychiatry, Sungkyunkwan University School of Medicine, Samsung Medical Center, Seoul, Korea
}

\begin{abstract}
Objective: We investigated the defense mechanisms and circadian preferences of a community sample. Methods: In total, 207 communitydwelling adults (122 males and 85 females, mean age 42.0 \pm 8.92 years) were recruited. All participants completed the Morningness-Eveningness Questionnaire (MEQ) to assess diurnal preference and the Defense Style Questionnaire (DSQ) to assess psychological defense mechanisms. The association between MEQ and the DSQ scores was determined after controlling for age. Results: The MEQ score was negatively correlated with an immature defense style $(r=-0.23, p<0.01)$ and a self-inhibiting defense style $(r=-0.14, p<0.05)$. In female participants, the MEQ score was negatively correlated with splitting $(r=-0.37, p<0.01)$ and consumption $(r=-0.23, p<0.05)$. The MEQ score was negatively correlated with fantasy in male participants $(r=-0.24, p<0.01)$. Conclusion: Eveningness was associated with an immature or self-inhibiting defense style. The association between eveningness and immature defense was affected by sex. The current results suggest that defense style is associated with diurnal preference in community-dwelling adults and indicate that this association differs between males and females. The current study suggests that circadian preference is associated with a specific defense mechanism, which may be affected by sex.
\end{abstract}

Key Words: Morningness-eveningness; Defense; Personality; Sex; Community

Received: February 8, 2019 Accepted: March 11, 2019

Corresponding author: Seog Ju Kim, MD, PhD, Department of Psychiatry, Sungkyunkwan University School of Medicine, Samsung Medical Center, 81 Ilwon-ro, Gangnam-gu, Seoul 06351, Korea.

Tel: 82-2-3410-3583, Fax: 82-2-3410-0050, E-mail: ksj7126@skku.edu

(a) This is an Open Access article distributed under the terms of the Creative Commons Attribution Non-Commercial License (https://creativecommons.org/licenses/by$\mathrm{nc} / 4.0)$ which permits unrestricted non-commercial use, distribution, and reproduction in any medium, provided the original work is properly cited.

\section{INTRODUCTION}

Morningness-eveningness is the dimension most commonly used to describe individual differences in circadian preference [1] People with more morningness have usually been described as "larks" who get up early, go to bed early, perform mentally and physically best in the morning hours, and are characterized as morning types. In contrast, people with more eveningness have usually been described as "owls" who prefer to stay out late, get up at a later time, perform best in the evening, and are characterized as evening types [2]. Morningness-eveningness has been considered an indicator of individual differences in circadian phase position and has been reported to be influenced by a variety of factors, such as age, sex, culture, and genetic profile [3]. Socioenvironmental factors, such as social schedule or sunlight exposure, also affect circadian rhythms [4].
Several previous studies on morningness-eveningness have reported an association between diurnal preferences and personality traits. Eveningness has been reported to be associated with high novelty-seeking, high harm-avoidance and low self-directedness as measured by the Temperament and Character Inventory [5]. Eveningness is related to a neurotic and extroverted personality, whereas morningness is related to agreeableness and conscientiousness [6-9].

"Defense mechanism" is a psychodynamic concept that refers to an unconscious mental process that protects the self against the anxiety arising from a psychic conflicts [10-12]. Defense mechanisms are associated with many personality traits, psychological characteristics, and types of psychopathology. An immature defense style has been associated with alexithymia [13], insecure attachment [14], distancing/avoidance coping strategies [15], personality disorder traits [16], or deliberate self-harm [17]. An 
immature defense mechanism is more commonly found in those with anxiety, depression, and eating problems [16,18,19].

Although both morningness-eveningness and defense mechanisms have been associated with personality traits or psychopathology, only one study has investigated the association between morningness-eveningness and defense mechanisms. A study of 148 medical school students reported that higher consumption and lower sublimation are associated with eveningness [20]. However, the age and the socio-cultural environment of the participants in that study are not generalizable to community subjects of various ages. Furthermore, morningness-eveningness is significantly affected by age [21], as the circadian phase advances with the aging process after adolescence. Therefore, we need to study the association between morningness-eveningness and defense mechanisms in a community sample with a wide age range. Additionally, the associations of psychiatric variables with morningness-eveningness can differ based on sex [22].

The objective of this study was to explore the associations between morningness-eveningness and defense mechanisms in a community-dwelling Korean sample of male and female with a wide age range. Our hypothesis was that eveningness is associated with immature personality traits and that this association differs by sex.

\section{METHODS}

\section{Participants}

Participants ( $\geq 19$ years of age) were recruited from apartment buildings, churches, and public health centers and hospitals in Incheon Province, Republic of Korea, by advertisement. In total, 207 participants (122 males and 85 females, mean age $42.0 \pm 8.92$ years) were recruited. No significant difference in age was observed between male and female participants (male: $43.1 \pm 8.69$; female $40.41 \pm 9.06$ years). The study protocol was approved by the Institutional Review Board of Gachon University of Medicine and Science, and all subjects provided written informed consent.

\section{Instruments}

The Korean version of the Horne and Östberg MorningnessEveningness Questionnaire (MEQ) was used to measure the morningness-eveningness preference of the subjects [23]. The MEQ consists of 19 items, with a higher MEQ score reflecting greater morningness. The reliability and validity of the Korean version of MEQ have been confirmed to be acceptable.

The Korean version of the Defense Style Questionnaire (DSQ) was used to assess subjects' defense styles [24]. The DSQ is a selfadministered questionnaire evaluating 16 defense styles categorized into four groups: 1) immature defenses: acting out, fantasy, projection, splitting, passive aggression, and consumption; 2) adaptive defenses: sublimation, humor, denial, and omnipotence; 3) selfinhibiting defenses: suppression, withdrawal, undoing, and reaction formation; 4) conflict-avoiding defenses: resignation and isolation.

\section{Statistical analysis}

The independent t-test was employed to compare continuous data on demographic variables, whereas the chi-square test was used to compare categorical data. The association between the MEQ score and each defense score on the DSQ was determined by Pearson's correlation analysis. As the MEQ is closely related to age [21], a partial correlation was used after controlling for age. Additionally, as studies have reported sex differences in circadian preference and defense styles, the correlation analyses were performed separately by sex. All statistical analyses were performed using SPSS version 22 software (IBM Corp., Armonk, NY, USA).

\section{RESULTS}

The DSQ and MEQ scores of all participants are presented in Table 1. The differences in the DSQ and MEQ scores between male and female participants are also presented in Table 1. The mean MEQ score was significantly higher in male than in female participants $(\mathrm{t}=2.15, \mathrm{p}=0.019)$.

Female participants obtained significantly higher scores for immature defenses than did male participants $(\mathrm{t}=-3.85, \mathrm{p}<0.001)$. Among immature defense styles, females scored higher on acting out $(\mathrm{t}=-2.87, \mathrm{p}<0.01)$, projection $(\mathrm{t}=-1.98, \mathrm{p}=0.049)$, fantasy $(\mathrm{t}=3.61$, $\mathrm{p}<0.001)$, and consumption ( $\mathrm{t}=-5.26, \mathrm{p}<0.001)$. Female participants also obtained higher scores for undoing $(\mathrm{t}=-3.32, \mathrm{p}=0.001)$. Male participants had higher scores for sublimation $(\mathrm{t}=2.23, \mathrm{p}=0.027)$.

The overall results revealed significant correlations between the MEQ and specific defense styles on the DSQ after controlling for age. The MEQ score was negatively correlated with an immature defense style $(\mathrm{r}=-0.23, \mathrm{p}<0.01)$ and a self-inhibiting defense style $(\mathrm{r}=-0.14, \mathrm{p}<0.05)$ after controlling for age. Among the immature defense styles, acting out $(\mathrm{r}=-0.15, \mathrm{p}<0.05)$, splitting $(\mathrm{r}=$ $-0.19, \mathrm{p}<0.01)$, fantasy $(\mathrm{r}=-0.22, \mathrm{p}<0.01)$, and consumption $(\mathrm{r}=$ $-0.18, p<0.05)$ were negatively correlated with the MEQ score after controlling for age. Withdrawal $(\mathrm{r}=-0.22, \mathrm{p}<0.01)$ and resignation $(\mathrm{r}=-0.15, \mathrm{p}<0.05)$ were also negatively correlated with the MEQ score after controlling for age (Table 2).

Differences in the associations between the MEQ and DSQ were observed between males and females. The significant association between the MEQ and the immature defense style was found only in females after controlling for age $(r=-0.26, p<0.05)$. Splitting $(\mathrm{r}=-0.37, \mathrm{p}<0.01)$ and consumption $(\mathrm{r}=-0.23, \mathrm{p}<0.05)$ were negatively correlated with the MEQ score in females, but not in males. In contrast, fantasy was correlated with the MEQ score after controlling for age only in males $(r=-0.24, p<0.01)$. Withdrawal was negatively correlated with the MEQ score in both males $(\mathrm{r}=-0.19, \mathrm{p}<0.05)$ and females $(\mathrm{r}=-0.28, \mathrm{p}<0.01)$ (Table 2).

\section{DISCUSSION}

In the current study, we found that eveningness was associated with immature and self-inhibiting defense styles in communitydwelling adults. We also found a sex difference in the association 
Table 1. Differences in morningness-eveningeness and defense mechanisms between males and females

\begin{tabular}{|c|c|c|c|c|c|}
\hline & Total $(n=207)$ & Male $(n=122)$ & Female $(n=85)$ & t-value & p-value \\
\hline Age (yr) & $42.0 \pm 8.92$ & $43.1 \pm 8.69$ & $40.41 \pm 9.06$ & $2.15^{*}$ & 0.033 \\
\hline \multicolumn{6}{|l|}{ Morningness-Eveningness } \\
\hline MEQ score & $49.89 \pm 8.48$ & $51.08 \pm 7.58$ & $48.17 \pm 9.41$ & $2.36^{*}$ & 0.019 \\
\hline \multicolumn{6}{|l|}{ Defense mechanisms } \\
\hline Immature defense style & $3.37 \pm 0.74$ & $3.21 \pm 0.70$ & $3.60 \pm 0.73$ & $-3.85^{* * *}$ & 0.000 \\
\hline Acting out & $3.45 \pm 1.09$ & $3.27 \pm 1.04$ & $3.71 \pm 1.12$ & $-2.87^{* *}$ & 0.005 \\
\hline Projection & $2.93 \pm 0.85$ & $2.83 \pm 0.84$ & $3.07 \pm 0.85$ & $-1.98^{*}$ & 0.049 \\
\hline Splitting & $3.86 \pm 0.91$ & $3.83 \pm 0.97$ & $3.83 \pm 0.97$ & -0.50 & 0.621 \\
\hline Fantasy & $3.22 \pm 1.33$ & $2.95 \pm 1.23$ & $3.61 \pm 1.39$ & $-3.61^{* * *}$ & 0.000 \\
\hline Passive aggression & $3.38 \pm 0.94$ & $3.32 \pm 0.96$ & $3.46 \pm 0.92$ & -1.05 & 0.295 \\
\hline Consumption & $3.40 \pm 1.13$ & $3.07 \pm 1.07$ & $3.87 \pm 1.05$ & $-5.26^{* * *}$ & 0.000 \\
\hline Adaptive defense style & $3.56 \pm 0.77$ & $3.50 \pm 0.77$ & $3.55 \pm 0.76$ & 0.14 & 0.890 \\
\hline Denial & $3.81 \pm 0.94$ & $3.82 \pm 1.00$ & $3.80 \pm 0.87$ & 0.20 & 0.844 \\
\hline Humor & $3.62 \pm 1.36$ & $3.56 \pm 1.32$ & $3.71 \pm 1.42$ & -0.76 & 0.446 \\
\hline Omnipotence & $3.26 \pm 1.01$ & $3.20 \pm 0.98$ & $3.33 \pm 1.06$ & -0.90 & 0.368 \\
\hline Sublimation & $3.54 \pm 0.99$ & $3.67 \pm 1.04$ & $3.36 \pm 0.89$ & $2.23^{*}$ & 0.027 \\
\hline Self-inhibiting defense style & $3.91 \pm 0.70$ & $3.90 \pm 0.66$ & $3.94 \pm 0.77$ & -0.40 & 0.688 \\
\hline Suppression & $4.12 \pm 0.90$ & $4.16 \pm 0.86$ & $4.07 \pm 0.97$ & 0.70 & 0.483 \\
\hline Reaction formation & $3.47 \pm 1.05$ & $3.48 \pm 1.06$ & $3.45 \pm 1.04$ & 0.17 & 0.865 \\
\hline Withdrawal & $4.29 \pm 1.34$ & $4.36 \pm 1.38$ & $4.20 \pm 1.30$ & 0.87 & 0.387 \\
\hline Undoing & $3.78 \pm 0.96$ & $3.60 \pm 0.94$ & $4.04 \pm 0.94$ & $-3.32^{* *}$ & 0.001 \\
\hline Conflict-avoiding defense style & $3.08 \pm 0.94$ & $3.01 \pm 0.93$ & $3.17 \pm 0.96$ & -1.22 & 0.223 \\
\hline Isolation & $2.95 \pm 1.05$ & $2.87 \pm 1.09$ & $3.05 \pm 0.98$ & -1.26 & 0.210 \\
\hline Resignation & $3.21 \pm 1.13$ & $3.15 \pm 1.12$ & $3.29 \pm 1.13$ & -0.88 & 0.381 \\
\hline
\end{tabular}

Variables are presented as mean \pm SD. ${ }^{*} \mathrm{p}<0.05,{ }^{* *} \mathrm{p}<0.01,{ }^{* * *} \mathrm{p}<0.001$. MEQ: Morningness-Eveningness Questionnaire.

Table 2. Association between morningness-eveningness and defense mechanisms controlling for age

\begin{tabular}{|c|c|c|c|}
\hline \multirow{2}{*}{ Defense mechanisms } & \multicolumn{3}{|c|}{ MEQ } \\
\hline & Total $(\mathrm{n}=207)$ & Male $(n=122)$ & Female $(\mathrm{n}=85)$ \\
\hline Immature defense style & $-0.23^{* *}$ & -0.16 & $-0.26^{*}$ \\
\hline Acting out & $-0.15^{*}$ & -0.13 & -0.12 \\
\hline Projection & -0.14 & -0.12 & -0.13 \\
\hline Splitting & $-0.19^{* *}$ & -0.03 & $-0.37^{* *}$ \\
\hline Fantasy & $-0.22^{* *}$ & $-0.24^{* *}$ & -0.13 \\
\hline Passive aggression & -0.10 & -0.02 & -0.20 \\
\hline Consumption & $-0.18^{*}$ & -0.09 & $-0.23^{*}$ \\
\hline Adaptive defense style & 0.05 & 0.04 & 0.04 \\
\hline Denial & 0.07 & 0.07 & 0.05 \\
\hline Humor & 0.03 & 0.05 & -0.03 \\
\hline Omnipotence & 0.00 & -0.01 & 0.00 \\
\hline Sublimation & 0.06 & -0.02 & 0.11 \\
\hline Self-inhibiting defense style & $-0.14^{*}$ & -0.11 & -0.18 \\
\hline Suppression & -0.13 & -0.11 & -0.16 \\
\hline Reaction formation & 0.04 & 0.00 & 0.05 \\
\hline Withdrawal & $-0.22^{* *}$ & $-0.19^{*}$ & $-0.28^{* *}$ \\
\hline Undoing & -0.02 & 0.08 & -0.07 \\
\hline Conflict-avoiding defense style & -0.12 & -0.06 & -0.15 \\
\hline Isolation & -0.05 & 0.01 & -0.09 \\
\hline Resignation & $-0.15^{*}$ & -0.11 & -0.18 \\
\hline
\end{tabular}

${ }^{*} \mathrm{p}<0.05,{ }^{* *} \mathrm{p}<0.01,{ }^{* * *} \mathrm{p}<0.001$. MEQ: Morningness-Eveningness Questionnaire. 
between eveningness and defense mechanisms. No previous studies have been performed on the association between morningness-eveningness and defense mechanisms in communitydwelling adults.

Consistent with our first hypothesis, eveningness was associated with the immature defense styles of acting out, splitting, fantasy, and consumption. This finding is consistent with a previous report on medical students [20]. The immature defense style is associated with inappropriate coping with impulses or drives [24]. Eveningness has been associated with difficulty with impulse control or the regulation of negative emotions [25-27]. The immature defense style of adults with eveningness may induce emotional instability or impulse control problems.

The current study also found that eveningness was associated with the withdrawal and the resignation. Both defense styles are related to avoidance from an emotionally challenging environment or interpersonal relationship. People with withdrawal or resignation may prefer nighttime activity as this involves reduced external or interpersonal stimuli. Eveningness was associated with less agreeableness in another study [28], which supports the association of eveningness with social and emotional withdrawal.

Consistent with our second hypothesis, there were sex differences in the association between defense style and morningnesseveningness. Splitting and consumption were associated with eveningness only in female adults. Splitting, which is a defense mechanism whereby the self and/or others are experienced as either all good or all bad, is associated with unstable interpersonal relationships and/or borderline personality disorder. The sleepwake cycle of those with borderline personality disorder is reportedly delayed [29]. Consumption, which is a defense mechanism whereby food, water, or alcohol is consumed to cope with stress, is associated with problematic eating or drinking. Eveningness has also been reported to be associated with emotional eating [30] or the consumption of alcohol or caffeine [31]. The prominent association between eveningness and splitting/consumption in females might be associated with the higher prevalence of borderline personality disorder or emotional eating in females than in males. Alternatively, evening-type females might ruminate over interpersonal conflicts or eat to reduce tension during the evening.

On the other hand, eveningness was associated with fantasy only in males. The use of fantasy as a defense mechanism usually involves autistic daydreaming or the creation of imaginary scenarios in which wishes and impulses are easily fulfilled. Autistic fantasy is reportedly associated with problematic internet use [32]. The prominent association between fantasy and eveningness may be mediated by night-time internet use or addictive gaming in males.

Several limitations of this study should be mentioned. First, our sample size was small. Future studies with larger sample sizes may be needed. Second, the cross-sectional design of the current study cannot address causal relationships between defense mechanisms and morningness-eveningness. Finally, the association between biological circadian rhythms and psychological defense mecha- nisms cannot be completely elucidated without measuring a biological indicator of circadian rhythms, such as melatonin, temperature, or cortisol.

In conclusion, the present study suggests that eveningness is associated with more immature and more self-inhibiting defense styles in community-dwelling adults. The current study also suggests that the association between specific defense mechanisms and diurnal preferences differs in males and females.

\section{Acknowledgments}

This study was financially supported by the Brain Research Program through the National Research Foundation of Korea, which is funded by the Ministry of Science, ICT \& Future Planning (NRF2016M3C7A1904338), and a National Research Foundation of Korea (NRF) grant funded by the Korean government (NRF-2016R1 A2B4011561).

\section{Conflicts of Interest}

The authors have no potential conflicts of interest to disclose.

\section{Author Contributions}

Conceptualization: Seog Ju Kim. Data curation: Jichul Kim, Somin Kim. Formal analysis: Jichul Kim, Somin Kim. Funding acquisition: Seog Ju Kim. Investigation: Jichul Kim, Seog Ju Kim. Methodology: Seog Ju Kim. Project administration: Seog Ju Kim. Resources: Seog Ju Kim. Supervision: Sehyun Jeon, Seog Ju Kim. Validation: Sehyun Jeon, Seog Ju Kim. Visualization: Jichul Kim. Writing_original draft: Jichul Kim. Writing_review \& editing: Somin Kim, Sehyun Jeon, Seog Ju Kim.

\section{ORCID iDs}

Seog Ju Kim (10)

https://orcid.org/0000-0003-2467-5451

Jichul Kim (10)

https://orcid.org/0000-0002-7660-4252

\section{REFERENCES}

1. Horne JA, Ostberg O. A self-assessment questionnaire to determine morningness-eveningness in human circadian rhythms. Int J Chronobiol 1976; 4:97-110.

2. Ishihara K, Miyasita A, Inugami M, Fukuda K, Miyata Y. Differences in sleepwake habits and EEG sleep variables between active morning and evening subjects. Sleep 1987;10:330-342.

3. Klei L, Reitz P, Miller M, Wood J, Maendel S, Gross D, et al. Heritability of morningness-eveningness and self-report sleep measures in a family-based sample of 521 hutterites. Chronobiol Int 2005;22:1041-1054.

4. Wittmann M, Dinich J, Merrow M, Roenneberg T. Social jetlag: misalignment of biological and social time. Chronobiol Int 2006;23:497-509.

5. Lee S, Park JE, Cho SJ, Cho IH, Lee YJ, Kim SJ. Association between morningness-eveningness and temperament and character in community-dwelling Korean adults. Asia Pac Psychiatry 2014;6:77-82.

6. Jackson LA., Gerard DA. Diurnal types, the "Big Five" personality factors, and other personal characteristics. J Soci Behav Pers 1996;11:273-283.

7. Randler C. Morningness-eveningness, sleep-wake variables and big five personality factors. Pers Individ Dif 2008;45:191-196.

8. Tonetti L, Fabbri M, Natale V. Relationship between circadian typology and big five personality domains. Chronobiol Int 2009;26:337-347. 
9. Adan A, Lachica J, Caci H, Natale V. Circadian typology and temperament and character personality dimensions. Chronobiol Int 2010;27:181-193.

10. Freud S. The neuro-psychoses of defense. London: Hogarth Press; 1894.

11. Freud S. Further remarks on the neuro-psychoses of defense. London: Hogarth Press; 1896.

12. VandenBos GR. APA dictionary of psychology. Washington: American Psychological Association; 2007.

13. Helmes E, McNeill PD, Holden RR, Jackson C. The construct of alexithymia: associations with defense mechanisms. J Clin Psychol 2008;64:318-331.

14. Costa RM, Brody S. Anxious and avoidant attachment, vibrator use, anal sex, and impaired vaginal orgasm. J Sex Med 2011;8:2493-2500.

15. Bouchard G, Theriault VJ. Defense mechanisms and coping strategies in conjugal relationships: an integration. Int J Psychol 2003;38:79-90.

16. Bond M. Empirical studies of defense style: relationships with psychopathology and change. Harv Rev Psychiatry 2004;12:263-278.

17. Brody S, Carson CM. Brief report: self-harm is associated with immature defense mechanisms but not substance use in a nonclinical Scottish adolescent sample. J Adolesc 2012;35:765-767.

18. Andrews G, Singh M, Bond M. The Defense Style Questionnaire. J Nerv Ment Dis 1993;181:246-256.

19. Blaya C, Dornelles M, Blaya R, Kipper L, Heldt E, Isolan L, et al. BrazilianPortuguese version of defensive style questionnaire- 40 for the assessment of defense mechanisms: construct validity study. Psychother Res 2007;17:261270.

20. Lee SJ, Park CS, Kim BJ, Lee CS, Cha BS, Kang H. Circadian preference and defense in medical students: greater consumption and lesser sublimation predicting eveningness. Sleep Med Psychophysiol 2013;20:82-87.

21. Kim SJ, Lee YJ, Kim H, Cho IH, Lee JY, Cho SJ. Age as a moderator of the association between depressive symptoms and morningness-eveningness. J Psychosom Res 2010;68:159-164.

22. Bae SM, Park JE, Lee YJ, Cho IH, Kim JH, Koh SH, et al. Gender difference in the association between adult attention deficit hyperactivity disorder symptoms and morningness-eveningness. Psychiatry Clin Neurosci 2010;64:649651.

23. Yu NJ, Shin S, Wnag SK. A study on the standardization of the Korean version of Horne and Ostberg's Morningness-Eveningness Questionnaire and on the sleep pattern. J Korean Neuropsychiatr Assoc 1995;34:642-656.

24. Cho SH. A validational study of Korean version of Defense Style Questionnaire. The Korean J Counsel Psychotherapy 1999;11:115-137.

25. Caci H, Robert P, Boyer P. Novelty seekers and impulsive subjects are low in morningness. Eur Psychiatry 2004;19:79-84.

26. Goldstein D, Hahn CS, Hasher L, Wiprzycka UJ, Zelazo PD. Time of day, intellectual performance, and behavioral problems in morning versus evening type adolescents: is there a synchrony effect? Pers Individ Dif 2007;42:431440.

27. Adan A, Natale V, Caci H, Prat G. Relationship between circadian typology and functional and dysfunctional impulsivity. Chronobiol Int 2010;27:606619.

28. Tsaousis I. Circadian preferences and personality traits: a meta-analysis. Eur J Pers 2010;24:356-373.

29. Huỳnh C, Guilé JM, Breton JJ, Godbout R. Sleep-wake patterns of adolescents with borderline personality disorder and bipolar disorder. Child Psychiatry Hum Dev 2016;47:202-214.

30. Konttinen H, Kronholm E, Partonen T, Kanerva N, Männistö S, Haukkala A. Morningness-eveningness, depressive symptoms, and emotional eating: a population-based study. Chronobiol Int 2014;31:554-563.

31. Giannotti F, Cortesi F, Sebastiani T, Ottaviano S. Circadian preference, sleep and daytime behaviour in adolescence. J Sleep Res 2002;11:191-199.

32. Waqas A, Rehman A, Malik A, Aftab R, Allah Yar A, Allah Yar A, et al. Exploring the association of ego defense mechanisms with problematic internet use in a Pakistani medical school. Psychiatry Res 2016;243:463-468. 